\title{
Evaluación de la discapacidad en población con trastornos mentales graves atendida en el Distrito Metropolitano de Quito (Ecuador)
}

\author{
Disability Assessment of Severe Mental \\ Disorders in Metropolitan District \\ of Quito-Ecuador Population
}

\author{
Marco Gamboa-Proaño, $\mathrm{PhD}(\mathrm{c})^{1}$ \\ Elvis Siprian Castro-Alzate, $\mathrm{PhD}^{2}$ \\ Claudio Bustos, $\mathrm{PhD}^{3}$ \\ Pamela Grandón F., PhD ${ }^{4}$ \\ Sandra Saldivia, $\mathrm{PhD}^{3 *}$
}

Avaliação da deficiência em população com transtornos mentais graves atendidas no distrito metropolitano de Quito-Equador

Recibido: 28 de enero de $2020 \cdot$ Aceptado: 7 de agosto de 2020

Doi: https://doi.org/10.12804/revistas.urosario.edu.co/revsalud/a.10149

Para citar este artículo: Gamboa-Proaño M, Castro Alzate ES, Bustos C, Grandón P, Saldivia S. Evaluación de la discapacidad en población con trastornos mentales graves atendida en el Distrito Metropolitano de Quito (Ecuador). Rev Cienc Salud. 2021;19(1):1-21. https://doi. org/10.12804/revistas.urosario.edu.co/revsalud/a.10149

1 Universidad Central del Ecuador. Programa de Doctorado en Psicología de la Universidad de Concepción (Chile).

2 Universidad del Valle (Colombia). Programa de Doctorado en Salud Mental de la Universidad de Concepción (Chile).

3 Departamento de Psiquiatría y Salud Mental, Universidad de Concepción (Chile)

4 Departamento de Psicología, Universidad de Concepción (Chile).

Marco Gamboa-Proaño: oRciD https://orcid.org/0000-0002-3971-5156

Elvis Siprian Castro-Alzate: oRCID https://orcid.org/0000-0003-2498-350X

Claudio Bustos: orciD https://orcid.org/0000-0003-3478-9858

Pamela Grandón F.: oRCID https://orcid.org/0000-0002-3952-1888

Sandra Saldivia: ORCID https://orcid.org/0000-0002-7594-7069

Autora de correspondencia: ssaldivi@udec.cl 


\section{Resumen}

Introducción: en Ecuador existen parámetros de calificación de discapacidad basados en la Clasificación Internacional del Funcionamiento de la Discapacidad y la Salud; sin embargo, los avances en investigación en el ámbito de la psiquiatría y la salud mental aún son escasos. Se empleó la Escala de Evaluación de Discapacidad de la Organización Mundial de la Salud para determinar el grado de discapacidad en población consultante. Materiales y métodos: estudio observacional, de corte transversal y correlacional. Usando un muestreo por conveniencia, se recogió información sobre discapacidad en una muestra de 297 adultos con trastornos mentales graves en contacto con servicios ambulatorios de psiquiatría. Se estimó un modelo de regresión lineal que determinó el aporte de variables sociodemográficas, clínicas y sociales. Resultados: el $89.2 \%$ de la muestra presentó algún grado de discapacidad. El modelo multivariado final para el total de la muestra incluyó las variables explicativas edad $(\beta=-0.234$ ), escolaridad ( $\beta=-0.552$ ), ser pensionado ( $\beta=39.44)$, presencia de episodio depresivo actual ( $\beta=21.654)$ y tener cuidador $(\beta=9.574)$, que explican el $37.46 \%$ de la discapacidad de las personas con trastornos mentales graves atendidas en servicios de psiquiatría y salud mental en Quito. Conclusión: la discapacidad en personas con trastornos mentales graves que están en contacto con servicios de salud de Quito (Ecuador) involucra cambios en el desempeño de actividades en escenarios familiares y comunitarios. Se identificó una proporción de discapacidad moderada y severa que demanda respuestas institucionales que incluyan el acompañamiento de cuidadores y aborden barreras actitudinales.

Palabras clave: trastornos mentales; esquizofrenia; trastorno depresivo mayor; trastorno bipolar; evaluación de la discapacidad.

\section{Abstract}

Introduction: In Ecuador, there are disability qualification parameters based on the International Classification of the Functioning of Disability and Health; however, advances in the research fields of psychiatry and mental health are still scarce. For this particular study, The World Health Organization Disability Assessment Scale was used to determine the degree of disability in the consulting population. Materials and methods: The study design was an observational, cross-sectional and correlational study. Using convenience sampling, disability information was collected from a study population of 297 adults with severe mental disorders who were in contact with outpatient psychiatric services. A linear regression model was used to determine the contribution of sociodemographic, clinical, and social variables. Results: It was observed that $89.2 \%$ of the sample presented some degree of disability. The final multivariate model for the total sample included the explanatory variables of age ( $\beta=-0.234)$, schooling ( $\beta=-0.552)$, being retired $(\beta=39.44)$, presence of current depressive episode $(\beta=21.654)$, and having a caregiver $(\beta=9.574)$, which explained $37.46 \%$ of disability in people with serious mental disorders who were treated in psychiatric and mental health services in Quito. Conclusion: Disabilities in people with severe mental disorders who are in contact with health services in Quito (Ecuador) may involve changes in the performance of activities among family and community settings. We identified a proportion of moderate and severe disabilities that demand institutional responses, which includes the accompaniment of caregivers and also addresses attitudinal barriers.

Keywords: Mental disorders; schizophrenia; major depressive disorder; bipolar disorder; disability assessment.

\section{Resumo}

Introdução: no Equador, existem parâmetros de qualificação de deficiência com base na Classificação Internacional do Funcionamento da Deficiência e da Saúde; no entanto, avanços em pesquisas no âmbito da psiquiatria e saúde mental ainda são escassos. Empregou-se a Escala de Avaliação da Deficiência da Organização Mundial da Saúde, para se determinar o grau de deficiência na população avaliada. Materiais 
e métodos: estudo observacional, de corte transversal e correlacional. Usando uma amostragem por conveniência coletou-se informação sobre deficiência em uma amostra de 297 adultos com transtornos mentais graves em contato com serviços ambulatórios em psiquiatria. Estimou-se um modelo de regressão linear que determinou a contribuição de variáveis sociodemográficas, clínicas e sociais. Resultados: 89.2\% da amostra apresentou algum grau de deficiência. $O$ modelo multivariado final para o total da amostra incluiu as variáveis explicativas idade $(\beta=-0,234)$, escolaridade $(\beta=-0,552)$, ser aposentado $(\beta=39,44)$, presença de episódio depressivo atual $(\beta=21,654)$ e, ter cuidador $(\beta=9,574)$, explicando $37,46 \%$ da deficiência em pessoas com transtornos mentais graves atendidas em serviços de psiquiatria e saúde mental em Quito. Conclusão: A deficiência em pessoas com transtornos mentais graves que estão em contato com serviços de saúde em Quito (Equador) envolve mudanças no desempenho de atividades em cenários familiares e comunitários. Identificou-se uma proporção de deficiência moderada e severa que demandam respostas institucionais que inclua o acompanhamento de cuidadores e abordem barreiras de atitude.

Palavras-chave: transtornos mentais; esquizofrenia; transtorno depressivo maior; transtorno bipolar; avaliação da deficiência.

\section{Introducción}

\section{$\mathrm{L}$} os trastornos mentales graves (TMG) han adquirido relevancia en la salud pública y en Mla investigación, porque generan una importante carga de morbilidad asociada a discapacidad $(1,2)$. Para la comprensión de los fenómenos vinculados a su complejidad, en las últimas décadas se han propuesto varias iniciativas que buscan desarrollar una definición consensuada. Distintas definiciones concuerdan en la multidimensionalidad del concepto $(3,4)$. Frances Allen, en uno de sus manifiestos, expone sobre la complejidad de llegar a un concepto claro de los TMG considerando que los criterios de normalidad y no normalidad se manejan desde parámetros subjetivos (5). Los trabajos previos de Slade y Powell se orientaron hacia una configuración que implica la interacción entre los factores de seguridad, apoyo —-formal e informal—, diagnóstico, discapacidad y duración (4); en la misma línea, Ruggieri y Leese plantearon tres dimensiones basadas en el diagnóstico, la duración y la discapacidad (3). En todas las definiciones de tmG, la discapacidad es uno de los criterios considerados.

Para la Organización Panamericana de la Salud (ops), los trastornos depresivos mayores, la esquizofrenia y los trastornos afectivos bipolares responden a los criterios para ser asumidos como TMG. Estas tres condiciones de salud aportan porcentajes de $3.35 \%, 0.86 \%$ y $0.59 \%$, respectivamente, a la carga específica de años de vida ajustados por discapacidad, para el conjunto de trastornos mentales, neurológicos y por consumo de sustancias en la región de las Américas (6), debido a que se han identificado entre las primeras causas de discapacidad global con implicaciones en los sistemas de salud, elevada carga económica para la familia y cambios en la vida cotidiana $(2,7)$. 
En Ecuador, la prevalencia por diagnóstico alcanza el 8.3\% para depresión, el 1.6\% para esquizofrenia y el $1.5 \%$ para trastornos bipolares, cifras similares a otros países de Suramérica (6).

La discapacidad, al ser considerada una dimensión de los TMG, se encuentra en un proceso histórico de producción intelectual en la región: reportes basados principalmente en estudios poblacionales en el contexto latinoamericano (8). Los esfuerzos recientes para llegar a acuerdos internacionales sobre el concepto de discapacidad se caracterizan por la transición, desde un modelo teórico basado en la presencia de consecuencias directas de una patología, donde las problemáticas son propias de la persona, a un modelo teórico, denominado relacional, resultante de la interacción entre la persona y el ambiente $(9,10)$.

Diversos autores han planteado las ventajas de incorporar este modelo de enfoque biopsicosocial (11-13), y usando la Clasificación Internacional del Funcionamiento, de la Discapacidad y de la Salud (CIF), es posible comprender la multidimensionalidad de los trastornos mentales. En la misma línea, se sugiere el empleo de la Escala de Evaluación de Discapacidad de la Organización Mundial de la Salud (whodas 2.0, por sus siglas en inglés), para comprender las implicaciones de estos trastornos en el funcionamiento social y ocupacional de las personas $(7,13,14)$.

Según el Ministerio de Salud Pública del Ecuador, la discapacidad mental existe cuando se presentan trastornos mentales con una alteración clínicamente significativa que refleje disfunción de procesos psicológicos, biológicos y sociales (15). De acuerdo con el Consejo Nacional para la Igualdad de Discapacidades del Ecuador, del total del registro nacional, el $5.13 \%$ corresponde a personas con discapacidad mental $(n=23928)$, y de estas el $20.0 \%$ tiene un grado de discapacidad severo o extremo, el 55.4\% son hombres, con predominio de edades comprendidas entre 30 y 64 años (53.5\%) (16). A partir de la implementación del Manual de calificación de la discapacidad, se incorporó el uso de parámetros de la cif en el reconocimiento de la discapacidad en la población ecuatoriana. Con la adopción de este sistema de clasificación se obtiene información de la discapacidad para elaborar un diagnóstico que incorpora la perspectiva de la relación entre el entorno y la persona, al comprender el funcionamiento y la discapacidad como una experiencia universal que trasciende el modelo rehabilitador centrado en las problemáticas directamente relacionadas con las personas con un diagnóstico clínico determinado $(15,17)$.

Diversos estudios en Latinoamérica han identificado la presencia de factores que determinan la ocurrencia de discapacidad en población con trastornos mentales con variables sociodemográficas, como el sexo y la ocupación; con variables clínicas, como la presencia de síntomas, el tiempo de evolución del diagnóstico clínico y la necesidad de manejo hospitalario, y con variables psicosociales, como la presencia de un cuidador o la necesidad de apoyo permanente, que incidirían como un factor de doble vía, por la presencia de alta dependencia o de barreras actitudinales, o la participación en actividades religiosas, que 
son un mecanismo para facilitar la participación en escenarios comunitarios (7,18,19,22). Tradicionalmente, los modelos empíricos de discapacidad obtenidos a partir de procesos de investigación se limitan a establecer la influencia de variables sin considerar la interdependencia y su capacidad explicativa.

Aunque la investigación sobre discapacidad se ha incrementado en los últimos años, aún es limitada, lo que puede obedecer a la complejidad de la cIF y a la atribución de una relación directa entre condiciones de salud física y discapacidad (23). Esta situación es similar en el contexto latinoamericano, donde existen algunos estudios recientes centrados en la necesidad de generar evidencia sobre la discapacidad en el ámbito de la psiquiatría y la salud mental (24).

En Ecuador, hasta la fecha, no se han desarrollado estudios que impliquen el uso de la WHODAs 2.0 en la evaluación del funcionamiento y la discapacidad en población con un diagnóstico de algún trastorno mental grave. Este trabajo pretende comprobar que la utilización de esta herramienta es válida en el medio local y que al contar con esta se tendrá un criterio técnico-científico en el momento de establecer porcentajes de discapacidad y, además, se podrá comparar con otros países de la región.

Esta investigación se llevó a cabo a través del modelo explicativo de la discapacidad en personas con diagnóstico de TMG, atendidas en hospitales de segundo y tercer nivel de la zona 9 del Distrito Metropolitano de Quito (Ecuador), considerando variables sociodemográficas, clínicas y psicosociales.

\section{Materiales y métodos}

C e llevó a cabo un estudio descriptivo, transversal y correlacional que recoge los datos de la muestra obtenida en Ecuador del estudio multicéntrico Comparación de modelos explicativos de discapacidad en población con trastornos mentales graves de tres países de Sudamérica, coordinado por un equipo interdisciplinario de investigadores de Colombia, Chile y Ecuador (24).

Se invitó a participar a personas de ambos sexos, con edades entre 18 y 65 años, con diagnóstico de TMG, incluyendo trastorno depresivo mayor, esquizofrenia y trastorno afectivo bipolar, de acuerdo con criterios la quinta edición del Manual diagnóstico y estadístico de los trastornos mentales, con evolución diagnóstica mayor o igual a dos años, clínicamente estables en el momento de la entrevista, que recibían atención ambulatoria en servicios de psiquiatría de hospitales generales y especializados de la zona 9 del Distrito Metropolitano de Quito (25). El único criterio de exclusión fue presentar deficiencias cognitivas, evaluadas con el Mini Mental State Examination, instrumento definido por el sistema de salud de Ecuador como método de tamizaje (26). 
Para definir el tamaño de la muestra se acudió a técnicas de un modelo estructural basado en una correlación mínima ( $\delta$ ) entre el número de variables latentes del instrumento $(k)$, teniendo en cuenta niveles de significancia ( $\alpha$ ) y el poder $(1-\beta)$, mediante la fórmula (27):

$$
n=f[k, \delta \mid \alpha, \beta]
$$

Los parámetros consideraron dos factores de la wHoDAs 2.0, correspondientes a las variables latentes limitaciones en la actividad y restricciones en la participación $(k=2)$; correlación mínima $(\delta=0,3)$; significancia $(\alpha=5 \%)$ y poder $(1-\beta=95 \%)(24)$. El tamaño mínimo requerido para la muestra fue de 274 personas.

Entre agosto y noviembre de 2018 se invitaron a participar a 393 personas, de las cuales 297 se incluyeron en el plan de análisis. De las 96 personas excluidas, 38 rechazaron participar, 3 presentaban diagnóstico menor a dos años y 55 presentaban deficiencia cognitiva, determinada a partir del tamizaje efectuado con el Mini-Mental Test (puntuación menor o igual a 22 puntos) (figura 1).

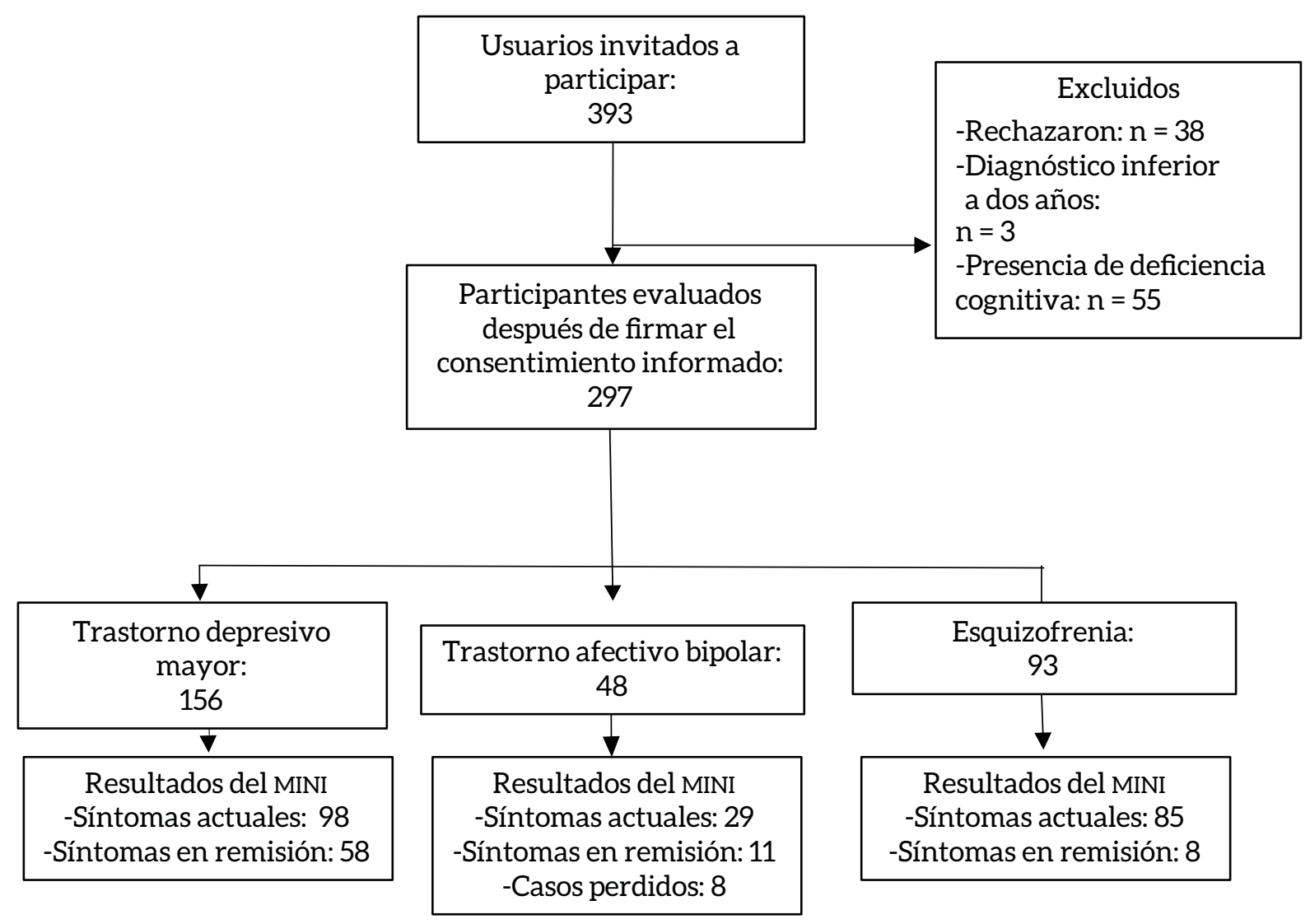

Figura 1. Diagrama de flujo del estudio

Los sujetos del estudio se reclutaron a partir del registro de atenciones del área de psiquiatría de los hospitales especializados Julio Endara, San Lázaro y del Hospital General Docente de Calderón. Los instrumentos los administraron tres estudiantes de último año de la carrera de Psicología de la Universidad Central del Ecuador, con entrenamiento previo 
en el protocolo, los instrumentos y la aplicación. La capacitación tuvo una duración de 40 horas y fue certificada por el Departamento de Psiquiatría y Salud Mental de la Universidad de Concepción (Chile) y la Facultad de Ciencias Psicológicas de la Universidad Central del Ecuador. Los participantes manifestaron de manera explícita su voluntad de participar en el estudio, mediante la firma de un consentimiento informado.

\section{Instrumentos}

WHODAS 2.0. Instrumento utilizado para evaluar la variable discapacidad. Se usó la versión heteroadministrada de 36 ítems. Puede ser administrado en múltiples condiciones de salud, incluidos los trastornos mentales, con opciones de respuesta tipo Likert que incluyen las categorías: ninguna [0], leve [1], moderada [2], severa [3] y extrema [4]. Este instrumento es producto de un consenso internacional y evalúa los componentes actividad y participación descritos en la CIF (28). Para la construcción de la versión original y la reducción del número de ítems se realizaron estudios de campo en Cuba, Perú y España, y para la validación en población general, el estudio de equivalencia cultural incluyó muestras de España, Colombia y México, que reportó altos índices de consistencia interna con a de Cronbach superiores a 0.9 y estabilidad temporal con coeficientes de correlación intraclase entre 0.69 y 0.89 (28). En Ecuador, fue validado en una muestra de personas con enfermedad de Parkinson (29).

La WHODAs 2.0 comprende seis dominios que permiten determinar cambios en el funcionamiento en actividades cotidianas (28). El primero abarca operaciones relacionadas con la cognición e involucra actividades de comunicación y pensamiento, específicamente concentración, memoria, resolución de problemas y aprendizaje. El segundo dominio corresponde a actividades relacionadas con el movimiento y la postura; involucra la experiencia de estar de pie y desplazamientos dentro del hogar y fuera de este. El tercero incluye el cuidado personal, indaga por actividades de higiene, alimentación y permanecer solo durante largos periodos. Estos tres dominios se agrupan en el factor denominado actividad $(28,30)$. El cuarto dominio corresponde a las relaciones con otras personas (familiares y desconocidas), incluidas las relaciones sexuales. El quinto incorpora las actividades de la vida diaria, incluidas las domésticas, las laborales y las educativas. El sexto dominio comprende la participación social, al indagar sobre la percepción que tiene la persona sobre los obstáculos del contexto físico y social para garantizar el desempeño de roles sociales y comunitarios (22). Estos tres dominios se agrupan en el factor denominado participación (28,30).

Las variables sociodemográficas y psicosociales se evaluaron a partir de la sección de datos sociodemográficos del instrumento.

Mini International Neuropsychiatric Interview (MINI). Instrumento usado para confirmar el diagnóstico de la historia clínica y para evaluar la presencia actual de sintomatología clínica. 
Específicamente, se emplearon los módulos de episodio depresivo mayor, trastornos psicóticos y trastornos maniacos/hipomaniacos, incluidos en la versión en español del instrumento (26).

Mini-Mental State Examination (Mini-Mental). Se aplicó para evaluar la presencia de deterioro cognitivo y riesgo de demencia. Se empleó la versión en español del instrumento, desarrollada por Folstein, Folstein y McHugh, que se ha usado en estudios previos en población ecuatoriana (31).

\section{Plan de análisis}

Se realizó un análisis univariado para una aproximación exploratoria a las variables sociodemográficas, clínicas y psicosociales; se establecieron frecuencias absolutas de los índices de discapacidad por dominios y por puntuación global, y se reportaron porcentajes y su respectivo intervalo de confianza al $95 \%$.

Para determinar la presencia de discapacidad en la muestra total, se ponderó la puntuación directa obtenida en la wHoDAs 2.0 a partir de la variable situación ocupación actual. Se ejecutó un análisis de regresión lineal múltiple, usando como variable dependiente la discapacidad, expresada en puntuaciones directas entre 0 y 100. Los puntos de corte para determinar las categorías de discapacidad se basaron en las recomendaciones de la cIF, de acuerdo con las siguientes puntuaciones directas: 0-4, ninguna discapacidad; 5-24, discapacidad leve; 25-49, discapacidad moderada; 50-95, discapacidad severa, y 96-100, discapacidad extrema.

El estudio original estableció una estructura general del aporte de cada una de las variables que permitió determinar un modelo multivariado usando análisis de bosques aleatorios y análisis Boruta $(24,32,33)$. Ambos procedimientos permitieron eliminar variables con pobre capacidad predictiva y estimar el aporte promedio de error de las variables seleccionadas, mediante permutación específica para la muestra de Ecuador.

La información se procesó en el paquete estadístico spss versión 23, compatible con Windows ${ }^{\circledR}$ y en lenguaje R. Se tuvieron en cuenta las consideraciones éticas estipuladas en la Declaración de Helsinki. El estudio fue aprobado por el Comité de Ética del Hospital Docente de Calderón (Ecuador) y por el Comité de Ética de la Universidad Central del Ecuador. Todos los participantes dieron su consentimiento informado mediante la firma del documento aprobado y certificado por el Comité de Ética de la Universidad Central del Ecuador. Se garantizaron los derechos de confidencialidad y la libertad de retirarse del estudio en cualquier momento sin consecuencias sobre su tratamiento. 


\section{Resultados}

a tabla 1 recoge los resultados de distribución de la muestra en sus variables sociodemo-
gráficas, clínicas y psicosociales.

Tabla 1. Características generales de la muestra

\begin{tabular}{|c|c|c|c|c|}
\hline Variables sociodemográficas & \multirow[t]{2}{*}{ n (297) } & \multirow[t]{2}{*}{$\%$} & \multicolumn{2}{|c|}{ Intervalo de confianza $95 \%$} \\
\hline Sexo & & & & \\
\hline Mujer & 199 & 67.0 & 61.3 & 72.3 \\
\hline Hombre & 98 & 33.0 & 27.7 & 38.6 \\
\hline \multicolumn{5}{|l|}{ Edad } \\
\hline Menor de 30 & 71 & 23.9 & 19.2 & 29.2 \\
\hline 30-39 años & 71 & 23.9 & 19.2 & 29.2 \\
\hline 40-49 años & 57 & 19.2 & 14.9 & 24.1 \\
\hline 50-59 años & 74 & 24.9 & 20.1 & 30.2 \\
\hline Mayor de 60 & 24 & 8.1 & 5.2 & 11.8 \\
\hline \multicolumn{5}{|l|}{ Escolaridad } \\
\hline Básica & 90 & 30.3 & 25.1 & 35.9 \\
\hline Media & 129 & 43.4 & 37.7 & 49.2 \\
\hline Superior & 68 & 22.9 & 18.2 & 28.1 \\
\hline Otra & 10 & 3.4 & 1.6 & 6.1 \\
\hline \multicolumn{5}{|l|}{ Estado civil } \\
\hline Soltero & 139 & 46.8 & 41.0 & 52.6 \\
\hline Casado-conviviente & 118 & 39.7 & 34.1 & 45.6 \\
\hline Separado-divorciado & 35 & 11.8 & 8.3 & 16.0 \\
\hline Viudo & 5 & 1.7 & 0.05 & 3.8 \\
\hline \multicolumn{5}{|l|}{ Ocupación actual } \\
\hline Desempleado & 65 & 21.9 & 17.3 & 27.0 \\
\hline Licencia médica & 3 & 1.0 & 0.2 & 2.9 \\
\hline Estudiante & 23 & 7.7 & 4.9 & 11.3 \\
\hline Actividades domésticas & 80 & 26.9 & 21.9 & 32.3 \\
\hline Asalariado-trabajador independiente & 125 & 42.1 & 36.4 & 47.9 \\
\hline \multicolumn{5}{|l|}{ Dependencia económica } \\
\hline Ingresos propios & 143 & 48.1 & 42.3 & 53.9 \\
\hline Familia & 238 & 80.1 & 75.1 & 84.5 \\
\hline
\end{tabular}

Continúa 


\section{Diagnóstico según historia clínica}

\begin{tabular}{|c|c|c|c|c|}
\hline TDM & 156 & 52.5 & 46.7 & 58.3 \\
\hline $\mathrm{TAB}$ & 48 & 23.5 & 12.2 & 20.9 \\
\hline EQZ & 93 & 37.2 & 26.1 & 36.9 \\
\hline \multicolumn{5}{|l|}{ Evolución diagnóstico } \\
\hline Menos de 10 años & 217 & 73.1 & 67.6 & 78.0 \\
\hline Entre 10 y 19 años & 56 & 18.9 & 14.6 & 23.8 \\
\hline Entre 20 y 29 años & 13 & 4.4 & 2.3 & 7.3 \\
\hline$\geq 30$ años & 11 & 3.7 & 1.8 & 6.5 \\
\hline \multicolumn{5}{|l|}{ Síntomas actuales (MINI) } \\
\hline ESR & 77 & 25.9 & 21.0 & 31.3 \\
\hline EPA & 85 & 28.6 & 23.5 & 34.1 \\
\hline EDA & 98 & 33.0 & 27.7 & 38.6 \\
\hline $\mathrm{EH} / \mathrm{MA}$ & 29 & 9.8 & 6.6 & 13.7 \\
\hline Casos perdidos & 8 & 2.7 & 0.1 & 5.2 \\
\hline \multicolumn{5}{|l|}{ Tipo tratamiento } \\
\hline Antipsicótico & 181 & 60.9 & 55.1 & 66.5 \\
\hline Estabilizador ánimo & 81 & 27.3 & 22.3 & 32.7 \\
\hline Antidepresivo & 155 & 52.2 & 46.3 & 57.9 \\
\hline Ansiolítico & 19 & 6.4 & 3.9 & 9.8 \\
\hline Variables psicosociales & $\mathbf{n}$ & $\%$ & Inter & anza $95 \%$ \\
\hline \multicolumn{5}{|l|}{ Presencia de cuidador } \\
\hline Sí & 65 & 21.9 & 17.3 & 27.0 \\
\hline \multicolumn{5}{|l|}{ Reporte de práctica religiosa } \\
\hline Sí & 171 & 57.6 & 51.7 & 63.3 \\
\hline \multicolumn{5}{|l|}{ Beneficiario de programas de apoyo } \\
\hline Educación & 5 & 1.7 & 0.5 & 3.8 \\
\hline Capacitación laboral & 4 & 1.3 & 0.4 & 3.4 \\
\hline Vivienda & 2 & 0.7 & 0.1 & 2.4 \\
\hline Recreación & 7 & 2.4 & 0.9 & 4.7 \\
\hline Subsidio & 4 & 1.3 & 0.4 & 3.4 \\
\hline Pensión & 34 & 11.4 & 8.1 & 15.6 \\
\hline
\end{tabular}




\section{Características del perfil de discapacidad en personas con TMG en Quito}

El 89.2 \% de la muestra presentó algún grado de discapacidad, con predominio de la discapacidad leve y moderada, en el $38.4 \%(n=114)$ y el $38 \%(n=113)$ de los casos, respectivamente; con un $12.8 \%(n=38)$ de discapacidad severa, mientras que el $10.8 \%(n=32)$ reportó ninguna discapacidad. Los perfiles de discapacidad se establecieron a partir de los siguientes dominios:

Dominio 1: cognición (comprensión y comunicación). Del total de la muestra, el 80.1\% $(n=238)$ presentó algún grado de discapacidad en esta dimensión. Según las categorías, se distribuyeron en discapacidad leve (25.6\%), discapacidad moderada (32.0\%) y discapacidad severa (22.6\%).

Dominio 2: movilidad en el entorno. El $62.6 \%$ de la muestra presentó algún grado de discapacidad en este dominio. El 30.6\% ( $n=91)$ tenía discapacidad leve, el $20.2 \%(n=60)$ presentó discapacidad moderada y el $11.8 \%(\mathrm{n}=35)$ evidenciaba discapacidad severa. Cabe señalar que el reporte de discapacidad en este dominio no implica la presencia de deficiencias físicas o sensoriales.

Dominio 3: autocuidado. Del total de la muestra, el 50.3\% $(\mathrm{n}=186)$ presentó algún grado de discapacidad en su autocuidado. Predomina la discapacidad leve, con $36.7 \%$ ( $n=109)$, seguida de discapacidad moderada $(17.8 \% ; n=53)$ y discapacidad severa $(4.7 \% ; n=14)$.

Dominio 4: relaciones personales. El $82.5 \%(n=245)$ de la muestra presentó algún grado de discapacidad en esta dimensión, lo que denota cambios en la forma o intensidad al establecer intercambio con otras personas, como miembros de la familia, la pareja, los amigos y desconocidos. En este dominio, la distribución de gravedad fue similar: un 25.6\% ( $n=76)$ reportó discapacidad leve; el 28.3\% ( $n=84)$, discapacidad moderada, y el $28.6 \%(n=85)$, discapacidad severa.

Dominio 5a: actividades domésticas. El 65.3\% (n=194) presentó algún grado de dificultad para realizar actividades domésticas, situación que implica cambios en el establecimiento de hábitos para ocuparse de responsabilidades inherentes al hogar. La distribución para cada categoría fue del $22.2 \%$ de discapacidad leve $(\mathrm{n}=66)$, del $24.2 \%(\mathrm{n}=72)$ de discapacidad moderada y del $18.9 \%(\mathrm{n}=56)$ de discapacidad severa.

Dominio 5b: trabajo y estudio. Este dominio incluyó al grupo de personas que en el momento de la evaluación refirió desempeñar un rol productivo como el trabajo (42.1\%, $\mathrm{n}=125)$ o estudio $(7.7 \%, \mathrm{n}=23)$. De estos, el $64.9 \%(\mathrm{n}=96)$ presentó algún grado de discapacidad. La distribución por gravedad fue de un $22.3 \%$ de discapacidad leve ( $\mathrm{n}=33$ ), un $27 \%$ de discapacidad moderada $(n=40)$ y un $15,5 \%$ de discapacidad severa $(n=23)$.

Dominio 6: participación social. De la muestra total, el $88.9 \%(\mathrm{n}=264)$ presentó algún grado de discapacidad para el desempeño de roles de acuerdo con las exigencias sociales en 
los diferentes contextos. La distribución mostró discapacidad leve en el $26.6 \%$ de los casos $(n=79)$, moderada en el $28.6 \%(n=85)$ y severa en el $33.7 \%(n=100)$.

\section{Análisis de regresión múltiple}

En la muestra de Ecuador, las variables explicativas del perfil de discapacidad incluyeron tres variables sociodemográficas, una variable clínica y una variable psicosocial. El modelo presenta un coeficiente de determinación de $0.3746\left(F[21.267]=7.616 ; p<0.001 ; \mathrm{R}^{2} \Delta=0.3254\right)$, que explica el $37.46 \%$ de la discapacidad de las personas con TMG de la muestra de Ecuador (tabla 2).

Tabla 2. Modelo de análisis multivariado para discapacidad en una muestra de personas con TMG atendidas en Quito (Ecuador)

\begin{tabular}{|c|c|c|c|c|}
\hline Variables $^{\mathrm{a}}$ & $\beta$ & Error estándar & Valor $t$ & $p$ \\
\hline Intercepto & 44.128 & 7.636 & 5.779 & $<0.001$ \\
\hline Edad & -0.234 & 0.099 & -2.350 & 0.0195 \\
\hline \multicolumn{5}{|l|}{ Sexo } \\
\hline Hombre & 3.104 & 2.430 & 1.277 & 0.202 \\
\hline Escolaridad & -0.552 & 0.264 & -2.085 & 0.038 \\
\hline \multicolumn{5}{|l|}{ Estado civil } \\
\hline Casado-pareja & -4.572 & 2.701 & -1.692 & 0.091 \\
\hline Separado-divorciado & 1.544 & 3.674 & 0.420 & 0.674 \\
\hline Viudo & 10.370 & 7.908 & 1.311 & 0.190 \\
\hline \multicolumn{5}{|l|}{ Situación laboral } \\
\hline Estudiante & -0.285 & 4.317 & -0.066 & 0.947 \\
\hline Actividades domésticas & -0.335 & 2.733 & -0.123 & 0.902 \\
\hline Pensionado & 39.440 & 18.963 & 2.080 & 0.034 \\
\hline Desempleado & -2.985 & 2.740 & -1.089 & 0.276 \\
\hline Licencia médica & -13.231 & 9.719 & -1.361 & 0.174 \\
\hline Evolución del diagnóstico (años) & 0.40 & 0.153 & 2.639 & 0.008 \\
\hline \multicolumn{5}{|l|}{ Síntomas actuales (MINI) } \\
\hline Episodio depresivo & 21.654 & 2.603 & 8.317 & $<0.001$ \\
\hline Episodio psicótico & 4.347 & 2.868 & 1.516 & 0.130 \\
\hline Episodio hipomaniaco/maniaco & 2.052 & 3.726 & 0.551 & 0.582 \\
\hline \multicolumn{5}{|l|}{ Programas de apoyo } \\
\hline Recreación & -3.418 & 8.368 & -0.409 & 0.683 \\
\hline Subsidio & 12.124 & 8.557 & 1.417 & 0.157 \\
\hline Pensión & 5.355 & 3.164 & 1.692 & 0.091 \\
\hline Otro & -12.661 & 5.289 & -2.394 & 0.017 \\
\hline
\end{tabular}




\begin{tabular}{lccccc}
\hline \multicolumn{1}{c}{ Variables $^{\mathrm{a}}$} & & $\boldsymbol{\beta}$ & Error estándar & Valor $t$ & $p$ \\
\hline Práctica religiosa & & & & & \\
& Sí & -3.673 & 2.119 & -1.733 & 0.084 \\
\hline Presencia de cuidador & & & & & \\
& Sí & 9.574 & 2.572 & 3.722 & 0.0002 \\
\hline
\end{tabular}

Error estándar residual: 16.16 en 267 grados de libertad.

$\mathrm{R}^{2}$ múltiple: 0.3746; $\mathrm{R}^{2}$ ajustado: 0.3254 .

Estadístico F: 7.616 en 21 y 267 grados de libertad, valor $p<0.001$.

a listado de comparadores: mujer, soltero, empleado/asalariado, síntomas en remisión.

Respecto de las variables sociodemográficas, por cada año de incremento en la edad, se presenta una reducción de 0.234 unidades en la carga de discapacidad; en el caso de la escolaridad, por cada año cursado y aprobado, se reduce la carga de discapacidad en 0.55 unidades; respecto de la situación laboral actual, en comparación con las personas empleadas, ser pensionado incrementa la carga de discapacidad en 39 unidades.

Al considerar las variables clínicas, la presencia de un episodio depresivo actual incrementa la carga de discapacidad en 21.7 unidades. Finalmente, en relación con las variables psicosociales, la necesidad de contar con un cuidador incrementa la carga de discapacidad en 9.6 unidades.

\section{Discusión}

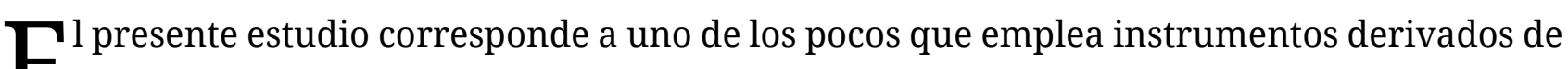
Ela cIF para evaluar discapacidad en población con tMg, en este caso usuarios de centros sanitarios del Distrito Metropolitano de Quito, lo que lo convierte en antecedente para la generación de evidencia sobre el tema en Latinoamérica.

La muestra estuvo conformada por personas con trastorno depresivo mayor (52.5\%), esquizofrenia ( $31.3 \%$ ) y trastorno afectivo bipolar (16.2\%). Estas entidades clínicas son parte de los TMG que la ops considera de gran interés, por el incremento en su incidencia y prevalencia global y por la necesidad de implementar políticas públicas orientadas a mejorar la calidad de vida de las personas. Cabe recalcar que Ecuador aún no cuenta con una política pública destinada a la salud mental que aborde estos puntos (6). En este sentido, el estudio realizado por Suárez-Escudero sostiene que cinco de cada diez causas de discapacidad corresponden a trastornos mentales como la depresión y la esquizofrenia, por lo que para comprender el impacto en la vida cotidiana es necesario determinar las características de la interacción entre la persona y el medio en el que se desenvuelve, que es un paso fundamental para disminuir el estigma y procurar la inclusión social (34). 
Otros estudios han demostrado que la discapacidad en población con TMG es frecuente y corresponde a una variable multidimensional en la que los factores personales y contextuales presentan diferentes ponderaciones en distintos modelos explicativos (1,2,7,18,35-38). Esta investigación encontró alta prevalencia de discapacidad, una situación notoria en la población consultante, al vincular el desempeño en la vida cotidiana con cada uno de los dominios evaluados mediante la wHODAs 2.0. Además, se encontró mayor afectación en los dominios cognición, relaciones sociales y participación social.

Se ha documentado que, en el caso de personas con TMG de larga evolución, la afectación funcional involucra un deterioro progresivo de funciones metales globales, entre las que se encuentran las funciones intelectuales, psicosociales globales y las funciones relacionadas con el funcionamiento intrapersonal $(6,8,23,37)$. Igualmente, desde la perspectiva relacional, el intercambio social con familiares, amigos y desconocidos puede verse afectado por tendencias hacia el autoaislamiento y la pérdida progresiva de roles sociales $(11,12,18)$.

Estudios realizados en Colombia, en los que se empleó la wHODAs 2.0, han mostrado resultados similares. En una muestra de personas con TMG se reportaron prevalencias del 46.2\% para discapacidad moderada y del 19.4\% para discapacidad severa (35). Igualmente, en una muestra de personas con TMG con antecedentes de intento de suicidio, se presentó una prevalencia del $95 \%$, con mayores frecuencias para discapacidad leve (41\%) y moderada (38\%) (22).

En el presente estudio se identificaron las variables que contribuyen en los diferentes índices de discapacidad que presentan las personas con TMG que participaron en esta investigación y que son atendidas en establecimientos de salud en Quito. Se incluyen a) variables sociodemográficas, específicamente edad, escolaridad y situación laboral-estar jubilado; b) una variable clínica, atribuida a la presencia de episodio depresivo actual, y c) una variable psicosocial, representada por la presencia de un cuidador. Esta última variable es comparable con los hallazgos del estudio comunitario de Zúñiga et al., mismo que concluye que la presencia de síntomas, la falta de cuidado en el hogar y la presencia del cuidador son factores que predicen el grado de funcionamiento y las necesidades sentidas por personas con TMG (39).

La edad y la escolaridad son variables que inciden en la ocurrencia de discapacidad y que la ops destaca en su informe de carga de discapacidad, junto con el fenómeno de envejecimiento poblacional y las limitadas oportunidades de mantenerse en el sistema educativo formal; esto hace que la carga de discapacidad en la región de las Américas se incremente (6). Los resultados que se obtuvieron en el presente estudio contrastan parcialmente con dichos antecedentes, puesto que, en el caso de la edad, la carga de discapacidad se reduce en 0.23 unidades a medida que la edad aumenta. Este particular se explicaría al considerar que los participantes de esta investigación presentaban una evolución diagnóstica de dos años y más, y se puede asumir que tener un tratamiento especializado contribuye a disminuir las 
barreras de la discapacidad, lo que mejora su calidad de vida desde el punto de vista de la función física (40). No se debe descartar que al ser usuarios de hospitales especializados o generales acuden a consulta para seguimiento o recibir las nuevas dosis de medicamentos, lo que nos hace presumir que existe una relación entre adherencia terapéutica y la presencia de un cuidador $(41,42)$.

Los resultados obtenidos con la muestra de sujetos de nuestro estudio reportan que el nivel de escolaridad se caracteriza por haber alcanzado la educación básica. El promedio de años cursados y aprobados fue de 10.6. Esta situación es similar a la de estudios de prevalencia en la región, en los que se concluye que la población con discapacidad alcanza un bajo nivel educacional (43). En este sentido, por cada año aprobado en educación formal, la carga de discapacidad se reduce en 0.55 unidades. La participación en medios sociales, el mantenimiento de destrezas cognitivas, el fortalecimiento de hábitos y rutinas favorecen la autonomía de las personas y, por ende, es factible la reducción objetiva de discapacidad en las personas con тмG $(44,45)$.

El rol productivo actual de la muestra se basa en actividades laborales independientes en el sector informal. Tal situación se explicaría por la escasa oferta laboral disponible para estos usuarios, y para la población en general, dadas las problemáticas económicas y sociales actuales en Ecuador, lo que refleja la influencia que sobre la pérdida de productividad y la capacidad laboral tienen —en el contexto latinoamericano-barreras actitudinales que limitan el acceso al trabajo digno, que reducen los ingresos y generan dependencia económica de las familias $(39,45,46)$. En la muestra estudiada, tener pensión por pérdida de la capacidad laboral (jubilación por discapacidad) es un factor explicativo de discapacidad en personas con TMG de Quito, situación que se exacerba cuando los síntomas depresivos están activos, lo que implica la disminución de experiencias sociales que involucran el desempeño en el rol laboral $(16,44)$.

A partir de las variables clínicas, se ha fundamentado que la discapacidad en los TMG se explica, principalmente, por alteraciones en funciones y estructuras del sistema nervioso. Esta condición es relevante, porque permite determinar que la presencia de síntomas psicóticos, maniacos o depresivos constituyen una variable con mayor capacidad explicativa de la carga de discapacidad (34). En la muestra de personas con TMG de Quito, los síntomas depresivos incrementan la carga de discapacidad en 21 unidades, en comparación con las personas que manifestaron presentar síntomas en resolución. Estos resultados son comparables con los hallazgos del estudio realizado en la ciudad de Cali (Colombia), en el que se identificó mayor probabilidad de carga de discapacidad en población con TMG con presencia de síntomas depresivos $(O R=4,47)(35)$. Al respecto, se ha establecido una relación entre los síntomas depresivos, el funcionamiento físico y la participación social, lo que influye directamente en la capacidad de disfrute y mantenimiento de hábitos y rutinas (47). Si una persona se encuentra con sintomatología actual, se incrementa la carga de discapacidad, y ello genera cambios en el desempeño 
de actividades con propósito en ámbitos familiares y comunitarios, especialmente si se trata de población con síntomas refractarios $(48,49)$.

Se ha documentado la relación directa entre la carga de discapacidad y la presencia de cuidador. En la muestra del presente estudio, la presencia de esta variable psicosocial incrementa la carga global de discapacidad en 9.6 unidades, resultado consistente con los hallazgos de Szkultecka-Dębek et al. y Caqueo-Urizar et al., quienes señalan que los TMG tienen un impacto directo en la calidad de vida de quien presenta el diagnóstico y de sus cuidadores $(19,37)$. En Colombia, los estudios de carga de cuidador y de carga de discapacidad han demostrado la relación entre estas dos variables, que se puede analizar de dos formas: la dependencia funcional del usuario, que genera la necesidad de acompañamiento por la desestructuración de hábitos y roles socialmente asociados, o los cuidadores, que sobreprotegen e infantilizan a los usuarios, lo que podría transformarse en una barrera para su funcionamiento $(20,35,50)$. Estos resultados constituyen una línea base para estudios posteriores que busquen comprender esta relación a partir de diseños longitudinales que permitan identificar una red causal y estimar una medida de asociación.

En conclusión, es preciso considerar que la muestra estudiada fue de personas que están en contacto con servicios de salud y asisten a consultas psiquiátricas; por lo tanto, tienen un seguimiento mínimo de dos años, lo que favorecería la disminución de discapacidad. Sin embargo, se identificó una considerable proporción de discapacidad moderada y severa, condición que exige respuestas a partir de planes y programas que impacten en estos usuarios, considerando el acompañamiento de cuidadores y abordaje de barreras actitudinales.

Estos resultados plantean el desafío de avanzar en investigaciones orientadas a la implementación y prueba de los mecanismos de evaluación de los sistemas de salud mental en Ecuador; más aún cuando se precisa medir el impacto de programas asistenciales sobre el funcionamiento social y ocupacional de la población consultante (45,51-53).

Adicionalmente, esta investigación genera resultados que permiten establecer mecanismos objetivos de evaluación de discapacidad, por lo que los retos a futuro se concentran en establecer observatorios de discapacidad que respondan a las orientaciones establecidas en el Manual de calificación de discapacidad para la población ecuatoriana (15).

\section{Limitaciones del estudio}

1 tratarse de una muestra por conveniencia obtenida de población consultante, los resulposible que la muestra esté sobrerrepresentada en función de la mayor participación de mujeres; sin embargo, esta condición obedece al perfil de usuario en el sistema sanitario ecuatoriano. 
Adicionalmente, estudios observacionales no permiten establecer relaciones causales, por lo que para dicho propósito se sugiere acudir a diseños que garanticen obtener medidas de asociación.

\section{Agradecimientos}

T os autores agradecen a las personas con TMG que participaron en el estudio, a las direcLtivas de los hospitales de la zona 9 del Distrito Metropolitano de Quito y a las psicólogas Janneth Jami, Norma Pelagallo y Mónica Guanoluisa, por su participación en la recolección de la información.

\section{Contribución de los autores}

W arco Gamboa-Proaño: adquisición, análisis e interpretación de los datos; planeación Elvis Siprian Castro-Alzate: concepción y diseño de la investigación; adquisición, análisis e interpretación de los datos; planeación del artículo o revisión de contenido intelectual importante; aprobación final de la versión para ser publicada, capacitación de evaluadores en Quito.

Claudio Bustos: análisis e interpretación de los datos; revisión de contenido intelectual importante; aprobación final de la versión para publicar.

Sandra Saldivia y Pamela Grandón Fernández: concepción y diseño; revisión de contenido intelectual importante; aprobación final de la versión para publicar.

\section{Financiación}

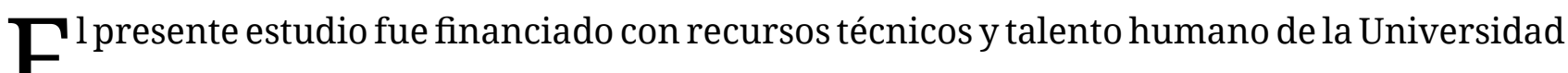
ECentral del Ecuador y de la Universidad del Valle (Colombia), y con recursos económicos de la Comisión Nacional de Ciencia y Tecnología de Chile (Conicyt, 2017) mediante la Beca de Doctorado Nacional, folio 21170614. 


\section{Conflicto de intereses}

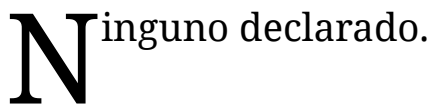

\section{Referencias}

1. Vigo D, Thornicroft G, Atun R. Estimating the true global burden of mental illness. Lancet. 2016;3(2):171-8. https://doi.org/10.1016/S2215-0366(15)00505-2

2. Vos T, Alemu A, Abbafati C, Abbas K, Hassen-Abate K, Abd-Allah F, et al. Global, regional, and national incidence, prevalence, and years lived with disability for 328 diseases and injuries for 195 countries, 1990-2016: a systematic analysis for the Global Burden of Disease Study 2016. Lancet. 2017;390:1211-59. https://doi.org/10.1016/S01406736(17)32154-2

3. Ruggieri M, Leese M, Thornicroft G, Bisoffi G, Tansella M. Definition and prevalence of severe and persistent mental illness. Br J Psychiatry. 2000;177(2):149-55. https://doi. org/10.1192/bjp.177.2.149

4. Slade M, Powell R, Strathdee G. Current approaches to identifying the severely mentally ill. Soc Psychiatry Psychiat Epidemiol. 1997;32(4):177-84. https://doi.org/10.1007/ bf00788236

5. Frances A. ¿Somos todos enfermos mentales? Manifiesto contra los abusos de la psiquiatría. Madrid: Ariel; 2014.

6. Organización Panamericana de la Salud. La carga de los trastornos mentales en la región de las Américas, 2018 [internet]. Washington D. C.: Organización; 2018. Disponible en: http://iris.paho.org/xmlui/bitstream/handle/123456789/49578/9789275320280_spa. pdf?sequence $=9 \&$ isAllowed $=y$

7. Hernández-Orduña O, Robles-García R, Martínez-López N, Muñoz-Toledo C, GonzálezSalas A, Cabello M, et al. WHodAs and the evaluation of disability among people with mental disorders with and without psychotic symptoms. Salud Mental. 2017;40(5):20917. https://doi.org/10.17711/SM.0185-3325.2017.027

8. Organización Panamericana de la Salud. Aplicación de la Clasificación Internacional del Funcionamiento, de la Discapacidad y de la Salud en estudios de prevalencia de discapacidad en las Américas [internet]. Washington D. C.: Organización; 2012. Disponible en: https://www.paho.org/arg/images/Gallery/Varias/informecif.pdf?ua=1

9. Peñas-Felizzola O. Referentes conceptuales para la comprensión de la discapacidad. Rev Fac Med. 2013;61(2):205-12.

10. Brandt E, Pope A. Enabling America: assessing the role of rehabilitation science and engineering [internet]. Washington, D. C.: National Academy Press; 1997. Disponible en: http://www.ncbi.nlm.nih.gov/books/NBK233578/pdf/Bookshelf_NBK233578.pdf

11. Vásquez-Barquero J. The incorporation of the disability construct as an independent axis in the DSM-V and ICD-11 diagnostic systems. World Psychiatry. 2009;8(2):92-4 
12. Gold L. DSM-5 and the assessment of functioning: the World Health Organization Disability Assessment Schedule 2.0 (wHodas 2.0). J Am Acad Psychiatry Law. 2014;42(2):173-81.

13. Guilera G, Gómez-Benito J, Pino O, Rojo E, Vieta E, Cuesta M, et al. Disability in bipolar I disorder: the 36-item World Health Organization Disability Assessment Schedule 2.0. J Affect Disord. 2015;174:353-60. https://doi.org/10.1016/j.jad.2014.12.028

14. Habtamu K, Alem A, Medhin G, Fekadu A, Dewey M, Prince M, et al. Validation of the World Health Organization Disability Assessment Schedule in people with severe mental disorders in rural Ethiopia. Health Qual Life Outcomes. 2017;15(64):2-11. https://doi. org/10.1186/s12955-017-0647-3

15. Ministerio de Salud Pública. Calificación de la discapacidad. Quito: Dirección Nacional de Normatización; 2018.

16. Servicio Nacional de Discapacidad (Senadis). Discapacidad y salud mental: una visión desde Senadis. Santiago: Ministerio de Desarrollo Social; 2016.

17. Querejeta M. Las herramientas del nuevo paradigma de la salud en el siglo xxI: CIE/CIF. Rev Esp Salud Publica. 2009;87:771-3.

18. Robles-García R, Medina-Dávalos R, Páez-Agraz F, Becerra-Rodríguez B. Evaluación de la funcionalidad, discapacidad y salud para la rehabilitación psicosocial de pacientes asilados por trastornos mentales graves. Salud Mental. 2010;33:67-75.

19. Caqueo-Urízar A, Rus-Calafell M, Craig T, Irarrazaval M, Urzúa A, Boyer l, et al. Schizophrenia: impact on family dynamics. Curr Psychiatry Rep. 2017;19:2-8. https:// doi.org/10.1007/s11920-017-0756-Z

20. Salazar-Torres L, Castro-Alzate E, Dávila-Vásquez P. Carga del cuidador en familias de personas con enfermedad mental vinculadas al programa de hospital de día de una institución de tercer nivel en Cali (Colombia). Rev Colomb Psiquiatr. 2019;48(2):88-95. https://doi.org/dx.doi.org/10.1016/j.rcp.2017.08.002

21. Gutiérrez-Maldonado J, Caqueo-Urízar A, Ferrer-García M, Fernández-Dávila P. Influencia de la percepción de apoyo y del funcionamiento social en la calidad de vida de pacientes con esquizofrenia y sus cuidadores. Psicothema. 2012;24(2):255-62.

22. Castro-Alzate E, Castillo-Martínez A. Factores relacionados con la carga de discapacidad en personas con intento de suicidio, atendidas en la red pública de servicios de salud de Santiago de Cali. Rev Colomb Psiquiatr. 2013;42(1):29-50.

23. Álvarez A. The application of the International Classification of Functioning, Disability, and Health in Psychiatry: possible reasons for the lack of implementation. Am J Phys Med Rehabil. 2012;91(13 suppl 1):S69-73. https://doi.org/10.1097/PHM.0b013e31823d4f1c

24. Castro-Alzate E, Saldivia S. Comparación de modelos explicativos del perfil de discapacidad en población con trastornos mentales graves de tres países de Sudamérica [informe de tesis doctoral]. Concepción (Chile): Universidad de Concepción; 2019.

25. American Psychiatric Association. Manual de diagnóstico y estadístico de los trastornos mentales. Madrid: Editorial Médica Panamericana; 2014.

26. Sheehan D, Lecrubier Y, Harnett-Sheehan K, Amorim P, Janavs J, Weiller E, et al. The Mini International Neuropsychiatric Interview (M.I.N.I.): The development and validation of a structured diagnostic psychiatric interview. J Clin Psychiatry. 1998;59(20):22-33. 
27. Westland J. Lower bounds on sample size in structural equation modeling. Electron Commer Res Appl. 2010;9:476-87. https://doi.org/doi.org/10.1016/j.elerap.2010.07.003

28. World Health Organization. Measuring health and disability: manual for wHo Disability Assessment Schedule wHODAs 2.0. Malta; 2010.

29. Serrano-Dueñas P, Mafla-Delgado D. Validación de la escala wHODAs 2.0 (World Health Organization Assessment Schedule) en pacientes con enfermedad de Parkinson que acuden a consulta externa de servicios de neurología del Hospital Carlos Andrade Marín (HсAм) durante el periodo de julio de 2013 a octubre de 2014 [informe de tesis de Especialidad Médica]. Quito: Pontificia Universidad Católica del Ecuador; 2014.

30. Federici S, Bracalenti M, Meloni F, Luciano J. World Health Organization disability assessment schedule 2.0: An international systematic review. Disabil Rehabil. 2016:1-34. https://doi.org/10.1080/09638288.2016.1223177

31. Estevez F, Abambari C, Atiencia R, Webster N. Deterioro cognitivo y riesgo de demencia: una realidad para el ecuador. Estudio de factores de riesgo en un grupo de pacientes jubilados del IEss en Cuenca en el Año 2013. Rev Ecuat Neurol. 2013;23(1):12-7.

32. Breiman L. Random forests. Mach Learn. 2001;45:5-32.

33. Kursa M, Rudnicki W. Feature selection with the Boruta Package. J Stat Softw. 2010;11(36):1-13.

34. Suárez-Escudero J. Discapacidad y neurociencias: la magnitud del déficit neurológico y neuropsiquiátrico. Acta Neurol Colomb. 2014;30(4):290-9.

35. Castro-Alzate E, Cardona-Marín L, Bustos C, Pacheco López R, Saldivia S. Modelo explicativo de discapacidad en población con trastornos mentales graves atendida en una institución especializada de la ciudad de Cali (Colombia). Rev Cienc Salud. 2019;17(3):6080. https://doi.org/10.12804/revistas.urosario.edu.co/revsalud/a.8366

36. Moro M, Frades B, Salazar J, Pena J, Asuero M. Aplicación de la Clasificación Internacional del Funcionamiento, de la Discapacidad y la Salud (CIF) de la oms para la evaluación de la discapacidad en pacientes con trastorno mental grave. Norte de Salud Mental. 2011;9(41):59-72.

37. Szkultecka-Dębek M, Miernik K, Stelmachowski J, Jakovljević M, Jukić V, Aadamsoo K, et al. Schizophrenia causes significant burden to patients' and caregivers' lives. Psychiatr Danub 2016;28(2):104-10.

38. Fekete C, Boldt C, Post M, Eriks-Hoogland I, Cieza A, Stucki G. How to measure what matters: development and application of guiding principles to select measurement instruments in an epidemiologic study on functioning. Am J Phys Med Rehabil. 2011;90(11):S2830. https://doi.org/10.1097/PHM.0b013e318230fe41

39. Zúñiga A, Navarro J, Lago P, Olivas F, Muray E, Crespo M. Evaluación de necesidades en pacientes psiquiátricos graves: un estudio comunitario. Actas Esp Psiquiatr. 2013;41(2):115-21.

40. Crespo-Facorro B, Bernardo M, Argimon J, Arrojo M, Bravo-Ortiz M, Cabrera-Cifuentes A, et al. Eficacia, eficiencia y efectividad en el tratamiento multidimensional de la esquizofrenia: proyecto Rethinking. Rev Psiquiatr Salud Ment. 2017;10(1):4-20. https://doi. org/doi.org/10.1016/j.rpsm.2016.09.001 
41. Galindo-Ocaña J, Ortiz-Camúñez MA, Gil-Navarro MV, Garrido Porras E, Bernabeu-Wittel M, Santos-Ramos B. La discapacidad como barrera a la adherencia terapéutica en pacientes pluripatológicos: papel del cuidador principal. Rev Clin Esp. 2010;210(5):221-6. https://doi.org/10.1016/j.rce.2009.11.015

42. Liberman R, Kopelwicz A. Un enfoque empírico de la recuperación de la esquizofrenia: definir la recuperación e identificar los factores que pueden facilitarla. Rehabil Psicosoc. 2004;1(1):12-29.

43. Neves-Silva P, Álvarez-Marín S. Estudio descriptivo de las características sociodemográficas de la discapacidad en América Latina. Ciênc Saúde Colet. 2014;19(12):4889-98.

44. Rodríguez-Ruiz E. El papel del to en la valoración funcional de personas con enfermedad mental. ToG (A Coruña). 2016;13(23):1-14.

45. Sango-Calo K, Merino-Salazar P. Empleo informal y depresión en el ecuador: resultados de la vi encuesta de condiciones de vida. Quito: Universidad Internacional sEK; 2019.

46. Edlund M, Wang J, Brown K, Forman-Hoffman V, Calvin S, Hedden S, et al. Which mental disorders are associated with the greatest impairment in functioning? Soc Psychiatry Psychiatr Epidemiol. 2018;53(11):1265-76. https://doi.org/10.1007/s00127-018-1554-6

47. Hudson J, Bower P, Archer J, P C. Does collaborative care improve social functioning in adults with depression? the application of the wно IcF framework and meta-analysis of outcomes. J Affect Disord. 2015;189:379-91. https://doi.org/10.1016/j.jad.2015.09.034

48. Tenorio-Martínez R, Lara-Muñoz M, Medina-Mora M. Measurement of problems in activities and participation in patients with anxiety, depression and schizophrenia using the IcF checklist. Soc Psychiatry Psychyatr Epidemiol. 2009;44(5):377-84. https://doi. org/10.1007/s00127-008-0449-3

49. Salvo L. Magnitud, impacto y estrategias de enfrentamiento de la depresión, con referencia a Chile. Rev Med Chile. 2014;142:1157-64. https://doi.org/10.4067/S003498872014000900010

50. Camargo-Rojas D, Castro-Alzate E, Hernández-Romero H, Maldonado-Salgado H, Cárdenas I, Carvajal L, et al. Conocimientos, actitudes y prácticas de personas con discapacidad, en procesos de inclusión social en el municipio de Madrid, Cundinamarca. Rev Cienc Salud. 2015;13(2):171-85. https://doi.org/10.12804/revsalud13.02.2015.04

51. Organización Panamericana de la Salud, Ministerio de Salud del Ecuador. Informe sobre el sistema de salud mental en Ecuador. Quito; 2008.

52. Reglamento a la ley orgánica de discapacidades, Decreto Ejecutivo 194 (2017).

53. Presidencia de la República del Ecuador. Decreto Ejecutivo 422, crea el bono Joaquín Gallegos Lara a favor de personas con discapacidad. Quito: Ecuador PdlRd, editor; 2010. 